\title{
Le contrôle des nuisances aéroportuaires, un modèle à reproduire pour le droit de l'environnement
}

\author{
Julien BÉTAILLE \\ Maître de conférences \\ à l'Université Toulouse 1 Capitole \\ IEJUC
}

L'étude du contrôle des nuisances aéroportuaires fournit une opportunité de se réjouir. Ce mécanisme peut être considéré comme étant plutôt efficace. Pour autant, cela n'est pas principalement le fait du rôle joué par le juge administratif mais tient davantage à l'architecture institutionnelle mise en place dans le domaine des transports aériens. Le caractère indépendant de l'autorité en charge du prononcé des sanctions est déterminant. Ce modèle pourrait être étendu dans d'autres domaines du droit de l'environnement.

Le contrôle des «nuisances aéroportuaires » ne porte pas seulement sur des nuisances au sens strict, mais il s'étend à quelques pollutions. Les pollutions et nuisances générées par les activités aéroportuaires sont en tout cas très variées même si, bien sûr, leur principal impact reste le bruit. Néanmoins, ces activités génèrent aussi de la pollution atmosphérique liée à la combustion du carburant des avions et des émissions de $\mathrm{CO} 2$, certaines pollutions de l'eau -notamment à cause des produits de dégivrage des avions tels que le glycol ou encore le risque d'inondation lié à l'imperméabilisation des sols aéroportuaires- ainsi qu'une certaine pollution lumineuse ${ }^{1}$. Ces pollutions et nuisances -

\footnotetext{
${ }^{1}$ V. Jacques Alain Bénisti et Christophe Bouillon, Rapport d'information sur les nuisances aéroportuaires, Assemblée nationale, 16 mars 2016, p. 38 et s.
} 
principalement les nuisances sonores et la pollution atmosphérique- sont contrôlées en raison des nombreux impacts qu'elles engendrent sur l'environnement et la santé ${ }^{2}$.

Afin de limiter ces impacts, le droit de l'environnement utilise des techniques devenues classiques. D'une part, il s'agit de réduire la vulnérabilité aux risques en maitrisant l'urbanisation au voisinage des aérodromes. C'est la loi du 11 juillet 1985, codifiée aux articles L. 112-3 et s. du Code de l'urbanisme, qui met en place un zonage du bruit à travers le plan d'exposition au bruit (PEB). D'autre part, il s'agit de réduire directement la source de pollutions et de nuisances. Selon un procédé classique -la police administrative spéciale-, une réglementation est mise en place et fixe des seuils de nuisance ou de pollution à ne pas dépasser ${ }^{3}$. Une autorité administrative est chargée de contrôler et de sanctionner le non-respect de ces règles. En l'occurrence, la direction générale de l'aviation civile (DGAC) et la gendarmerie des transports aériens (GTA) sont chargées de contrôler les compagnies aériennes et c'est ensuite l'Autorité de contrôle des nuisances aéroportuaires (ACNUSA) qui prononce des sanctions. L'action exercée par l'ACNUSA -à laquelle s'est peu intéressée la doctrine juridique ${ }^{4}$ - a par nature un impact direct sur le niveau des nuisances aéroportuaires, ce qui n'est pas le cas de la maîtrise de l'urbanisation ${ }^{5}$. Cela conduit à consacrer cette étude au

\footnotetext{
${ }^{2}$ Un récent rapport parlementaire a souligné les conséquences des nuisances aéroportuaires sur la santé (Jacques Alain Bénisti et Christophe Bouillon, ibidem, p. 49 et s.), principalement à cause du bruit. Au-delà de la santé, cela représente également des coûts sociaux importants (v. Jacques Alain Bénisti et Christophe Bouillon, ibidem, p. 59 et s.), des dépréciations immobilières et de la «polarisation sociale ». Les impacts sur la santé sont particulièrement soulignés par le Président de 1'ACNUSA : « en 2015, de nouvelles études ont confirmé que les nuisances aéroportuaires ont de graves conséquences sur la santé des riverains et des professionnels travaillant sur les plateformes ainsi que sur la qualité de l'environnement. Tout cela représente des coûts économiques et sociaux élevés, en grande partie cachés et mal évalués » (Victor Haïm, Rapport d'activités de l'ACNUSA 2015).

${ }^{3}$ Ces règles sont principalement dispersées entre le Code de l'environnement (pollution de l'air et de l'eau) et le Code de l'aviation civile (bruit).

4 Aucune étude ne porte spécifiquement sur l'ACNUSA, même si son existence est mentionnée et que son fonctionnement est décrit par des travaux plus généraux (v. par exemple Marianne Moliner-Dubost, « Bruit », Fasc. 380, JurisClasseur Administratif, 2012, $\mathrm{n}^{\circ} 26$ s. ; Véronique Jaworski, «Lutte contre le bruit », Fasc. 4600, JurisClasseur Environnement et Développement durable, 2007, $\mathrm{n}^{\circ} 228$; Michel Prieur et al., Droit de 1'environnement, Précis, Dalloz, 2016, $\left.\mathrm{n}^{\circ} 451\right)$.

${ }^{5}$ Celle-ci est largement étudiée : v. Fernand Bouyssou, « Le bruit dans le droit de l'urbanisme », Droit et Ville, 1980, $\mathrm{n}^{\circ} 10$, p. 51 ; Véronique Inserguet,
} 
contrôle des nuisances -entendu au sens large ${ }^{6}$ - opéré par l'ACNUSA ${ }^{7}$, cela d'autant plus qu'à l'examen, ce contrôle revêt un intérêt qui dépasse largement le domaine des transports aériens pour s'étendre au droit de l'environnement et au droit des autorités administratives indépendantes (AAI).

L'ACNUSA est historiquement la première AAI créée dans le domaine de l'environnement ${ }^{8}$. Elle exerce, depuis sa création en $1999^{9}$, plusieurs types de pouvoirs, dont celui de sanctionner les compagnies aériennes en raison des nuisances sonores et de la pollution atmosphérique parfois excessives qu'elles génèrent ${ }^{10}$. Les principaux manquements sanctionnés par l'ACNUSA sont

«Urbanisme au voisinage des aérodromes », actualisation par Julien Bétaille et Sophie de Fontaine, Jurisclasseur Collectivités territoriales, Fasc. 1178, 2015.

${ }^{6} \mathrm{Le}$ «contrôle » englobe ici tout le processus lié au prononcé et à l'exécution d'une sanction par l'ACNUSA.

${ }^{7}$ Cela conduit à laisser de côté un certain nombre d'éléments tout aussi digne d'intérêt. D'une part, la jurisprudence de la Cour européenne des droits de l'homme a porté à plusieurs reprises sur des nuisances aéroportuaires, notamment avec l'arrêt du 8 juillet 2003, Hatton, $n^{\circ}$ 36022/97. Sans développer ici le contenu de cette jurisprudence, on retiendra néanmoins à la suite de Marianne Moliner-Dubost que l'article 8 de la Convention européenne des droits de l'homme « ne confère qu'une protection aléatoire contre des nuisances extrêmes » («Réflexions environnementalistes sur la protection juridique du calme », RDP, 2012, p. 1145). D'autre part, les contentieux portant sur le projet d'aéroport de Notre-Dame-des-Landes attirent l'attention sur l'impact des aéroports sur l'environnement, notamment au niveau de leur emprise au sol et de l'éventuelle destruction des zones humides (v. notamment Agathe Van Lang, «Le projet d'aéroport de Notre-Dame-des-Landes à l'épreuve de la compensation écologique-et inversement », Droit administratif, 2013, ét. 16).

8 Sur les AAI environnementales, v. Michel Prieur et al., Droit de l'environnement, op. cit., p. 357 s.

9 L'ACNUSA trouve en réalité son origine dans le décret n ${ }^{\circ}$ 97-284 du 27 mars 1997 qui avait créé une institution indépendante chargée du contrôle des nuisances sonores de Roissy et Orly. La loi $n^{\circ} 99-588$ du 12 juillet 1999 a ensuite créé l'autorité de contrôle des nuisances aéroportuaires, en gardant le même acronyme. Depuis la loi Grenelle II du 12 juillet 2010, l'ACNUSA n'est plus focalisée principalement sur les nuisances sonores mais a également en charge toutes les autres pollutions ou nuisances aéroportuaires, notamment la pollution atmosphérique.

${ }^{10} \mathrm{Sa}$ composition et son fonctionnement sont régis par les articles L. 6361-1 s. du Code des transports. Les membres de l'ACNUSA, au nombre de 10, sont soit nommés par le ministre de l'écologie, le ministre de la santé et le ministre de l'aviation civile, soit désignés par le Sénat ou l'Assemblée nationale. Dans le cadre de la mission de sanction, 7 «membres associés » participent aux séances, sans pour autant avoir de pouvoir de décision. 
les suivants ${ }^{11}$. Tout d'abord, il s'agit du non-respect de l'obligation d'évoluer à l'intérieur du «volume de protection environnementale $(\mathrm{VPE})^{12}$ : le VPE est une enveloppe de trajectoire à l'intérieur de laquelle l'avion doit évoluer au décollage et à l'atterrissage. Cette obligation est parfois contournée par les compagnies aériennes, au prétexte de mauvaises conditions météo, dans le but d'effectuer des économies de carburant. Ensuite, les vols de nuit sont réglementés dans les différents aéroports. Ces règles ne sont parfois pas respectées en raison des divers retards accumulés par les compagnies pendant la journée. Enfin, les aéronefs font l'objet de normes techniques qui les classent en différentes catégories, selon le niveau de nuisances qu'ils génèrent.

La procédure de sanction suivie par l'ACNUSA est prévue aux articles L. 6312 et s. du Code des transports ${ }^{13}$. Elle débute par l'établissement d'un procès-verbal, soit par les agents de la DGAC, soit par ceux de la gendarmerie des transports aériens. Ces PV sont notifiés à la personne concernée et transmis à l'ACNUSA. Celle-ci procède ensuite à une instruction

11 L'article L. 6361-12 du Code des transports définit dans le détail les manquements qui peuvent être sanctionnés par l'ACNUSA. Ils concernent : «a) Des restrictions permanentes ou temporaires d'usage de certains types d'aéronefs en fonction de leurs émissions atmosphériques polluantes, de la classification acoustique, de leur capacité en sièges ou de leur masse maximale certifiée au décollage ; b) Des restrictions permanentes ou temporaires apportées à l'exercice de certaines activités en raison des nuisances environnementales qu'elles occasionnent ; c) Des procédures particulières de décollage ou d'atterrissage en vue de limiter les nuisances environnementales engendrées par ces phases de vol ; d) Des règles relatives aux essais moteurs; e) Des valeurs maximales de bruit ou d'émissions atmosphériques polluantes à ne pas dépasser ». Les restrictions d'exploitation auxquelles il est fait référence sont prévues aux articles R. 227-8 s. du Code de l'aviation civile.

${ }^{12}$ Le volume de protection environnementale définit « des enveloppes de trajectoires permettant d'encadrer, dans les plans horizontal et vertical, les procédures de départ initial et d'approche finale, en vue de limiter les dispersions de trajectoires » (Marianne Moliner-Dubost, « Bruit », Fasc. 380, JurisClasseur Administratif, 2012, $\mathrm{n}^{\circ}$ 169).

${ }^{13}$ Cette procédure a pour origine le décret $\mathrm{n}^{\circ}$ 97-534 du 27 mai 1997. Il met en place un système de sanctions administratives se substituant à des amendes contraventionnelles. La procédure est aujourd'hui encadrée par les articles L. 6361-12 s. du Code des transports et R. 227-1 s. du Code de l'aviation civile (décret $\mathrm{n}^{\circ} 2010-405$ du 27 avril 2010 relatif à la procédure devant l'ACNUSA statuant en matière de sanctions, JORF du 28 avril 2010). En complément, v. la délibération du 28 avril 2010 portant règlement intérieur de l'Autorité de contrôle des nuisances sonores aéroportuaires (JORF n 0101 du 30 avril 2010 ; NOR : ACNX1011604X). 
contradictoire, sous l'autorité d'un rapporteur permanent. Ce dernier notifie le dossier à la personne concernée, laquelle peut présenter des observations. A l'issue de l'instruction, le Président de l'ACNUSA peut classer l'affaire sans suite ${ }^{14}$. Dans le cas contraire, après avoir entendu le rapporteur et la personne concernée, l'autorité délibère sur la sanction à adopter ${ }^{15}$. La décision de sanction doit être motivée et fait l'objet d'un recours de pleine juridiction ${ }^{16}$. Le montant maximum des amendes est de 1500 euros lorsque l'auteur du manquement est une personne physique et de 20000 euros lorsqu'il s'agit d'une personne morale. Dans certains cas, ce montant peut être porté jusqu'à 40000 euros $^{17}$.

Ce dispositif -relativement sophistiqué- peut être replacé dans une perspective plus générale. En effet, le droit de l'environnement est un droit finaliste : il poursuit un objectif déterminé, la protection de l'environnement. Dès lors, son étude conduit naturellement à interroger les dispositifs mis en place sous l'angle de leur contribution à la protection de l'environnement, autrement dit en terme d'effectivité ${ }^{18}$. En réponse à cette interrogation, nous voudrions montrer que le système répressif mis en œuvre par l'ACNUSA peut être

\footnotetext{
${ }^{14}$ Un rapport parlementaire indique par erreur que «l'ACNUSA n'a pas la responsabilité de l'engagement des poursuites » (Jacques Alain Bénisti et Christophe Bouillon, Rapport d'information sur les nuisances aéroportuaires, op. cit., p. 124).

${ }^{15}$ Il existe sept «membres associés » qui participent à la séance mais pas aux délibérations et ils ne prennent pas part au vote. Avant la loi du 8 décembre 2009 relative à l'organisation et à la régulation des transports ferroviaires et portant diverses dispositions relatives aux transports, il existait la Commission nationale de prévention des nuisances. Cette commission a été supprimée et désormais, l'ACNUSA est assistée par sept membres associés. Parmi eux figurent notamment des représentants des compagnies aériennes, des associations de riverains et des associations de protection de l'environnement (article L. 6361-4 du Code des transports et l'arrêté du 25 mars 2016 portant nomination de ces membres).

${ }^{16}$ Article L. 6361-13 al. 2 du Code des transports.

${ }^{17}$ L'article L. 6361-13 du Code des transports dispose que «s'agissant des personnes morales, ce montant maximal est porté à $40000 €$ lorsque le manquement concerne : $1^{\circ}$ Les restrictions permanentes ou temporaires d'usage de certains types d'aéronefs en fonction de leurs émissions atmosphériques polluantes ou de la classification acoustique ; $2^{\circ}$ Les mesures de restriction des vols de nuit $\gg$.

${ }^{18}$ V. Julien Bétaille, Les conditions juridiques de l'effectivité de la norme en droit public interne, illustrations en droit de l'urbanisme et en droit de l'environnement, thèse, droit, 2012 ; «Répression et effectivité de la norme environnementale », Revue juridique de l'environnement, $\mathrm{n}^{\circ}$ spécial, 2014 , p. 47.
} 
considéré comme globalement satisfaisant et qu'à ce titre il pourrait être intéressant de s'en inspirer.

En particulier, le thème de ce colloque -dédié au juge administratif- nous invite à examiner quel est le rôle occupé par ce juge vis-à-vis de ce système de contrôle des nuisances. Celuici est en fait quasiment neutre du point de vue de l'effectivité. En effet, le contrôle qu'il exerce sur les décisions de sanction de l'ACNUSA ne semble ni trop faible ni trop poussé. Ainsi, s'il contrôle effectivement l'action de cette autorité, il lui laisse suffisamment de liberté pour qu'elle exerce son rôle de régulateur vis-à-vis des compagnies aériennes. On constate ainsi, en premier lieu, l'exercice d'un contrôle adapté par le juge administratif (I). Au-delà, qu'est-ce qui rend le contrôle des nuisances aéroportuaires globalement satisfaisant? Il nous semble que la réponse à cette question se trouve du côté de l'architecture institutionnelle mise en place dans ce domaine. Contrairement aux autres domaines du droit de l'environnement, le pouvoir de sanction a ici été confié à une autorité administrative « indépendante ». Cette indépendance rend possible un exercice impartial du pouvoir de sanction, ce qui constitue un facteur d'effectivité des sanctions. Dès lors, il s'agit de montrer, en second lieu, en quoi le recours à une autorité indépendante peut être considéré comme un modèle à reproduire (II).

\section{L'EXERCICE D'UN CONTRÔLE ADAPTÉ PAR LE JUGE ADMINISTRATIF}

L'examen de la jurisprudence montre que le contrôle exercé par le juge administratif sur le pouvoir de sanction de l'ACNUSA se révèle relativement neutre à l'égard de l'efficacité de ce pouvoir de sanction ${ }^{19}$. En effet, s'il apporte les garanties classiques dans ce domaine, il reste suffisamment libéral pour ne pas entraver excessivement l'action de l'ACNUSA.

En premier lieu, le juge ayant reconnu l'applicabilité du droit au procès équitable à la procédure de sanction des nuisances

\footnotetext{
${ }^{19}$ Cette étude est basée sur un examen aussi systématique que possible de la jurisprudence. Cela a été rendu possible par le faible nombre de décisions rendues par les juridictions administratives. Nous avons ainsi pu consulter toutes les décisions du Conseil d'Etat et des Cours administratives d'appel, ainsi que les jugements de tribunaux administratifs signalés dans les rapports d'activité annuels de l'ACNUSA.
} 
aéroportuaires, l'ACNUSA doit respecter une partie des garanties liées au droit au procès équitable, en particulier les droits de la défense (A). En second lieu, le contrôle de la proportionnalité des sanctions mérite une attention particulière dans la mesure où c'est lui qui est le plus susceptible d'impacter l'efficacité des sanctions prononcées par l'ACNUSA. La façon dont le juge administratif l'exerce nous semble plutôt adaptée (B).

\section{A. L'APPLICABILITE DES GARANTIES LIEES AU DROIT AU PROCES EQUITABLE}

La reconnaissance de l'applicabilité de l'article 6 de la Convention européenne des droits de l'homme a conduit à renforcer les garanties procédurales, même si plusieurs de ces garanties ressortaient déjà du droit interne. Ainsi, le juge contrôle le respect des droits de la défense tout au long de la procédure de sanction mise en œuvre par l'ACNUSA ${ }^{20}$, de même que le respect de l'obligation de motiver la décision de sanction $^{21}$ ou encore le respect du principe de non-cumul des sanctions selon lequel un même manquement ne peut donner lieu qu'à une seule sanction administrative, sauf si la loi en dispose autrement ${ }^{22}$. Très récemment, le Conseil d'Etat a eu l'occasion, à propos d'une affaire impliquant l'ACNUSA, de préciser le contenu du principe général du droit de non cumul des sanctions. Ainsi, « cette règle s'applique tant lorsque l'autorité avait initialement infligé une sanction que lorsqu'elle avait décidé de ne pas en infliger une $»^{23}$.

${ }^{20}$ CE, sect., 31 janvier 2007, Corsair, ${ }^{\circ}$ 290567, Revue de droit des transports, 2007, comm. 69, obs. Loïc Grard ; Environnement, 2007, comm. 64, obs. David Gillig ; CE, 19 janvier 2009, Société Air France, n 315886 ; CE, 4 décembre 2009, Société Air France, $\mathrm{n}^{\circ}$ 323501. Plus généralement, v. CC, 2 décembre 1976, n 76-70 DC ; RDP, 1978, p. 817, note Louis Favoreu ; CC, 17 janvier 1989, n 88-248 DC, § 29 ; RDP, 1989, p. 429, note Louis Favoreu ; CE, Ass., 26 octobre 1945, Aramu et a., rec., p. 213 ; Dalloz, 1946, p. 158, note Georges Morange ; CE, 5 mai 1944, Dame Veuve Trompier Gravier, rec., p. 133 ; RDP, 1944, p. 256, concl. Bernard Chénot, note Gaston Jèze. Les droits de la défense sont désormais garantis par les articles L. 122-1 s. et L. 211-2 du Code des relations entre le public et l'administration.

${ }^{21}$ CE, 7 mai 2008, Société Air France, n 305826.

${ }^{22}$ CE, 29 octobre 2009, Société Air France, $n^{\circ} 312822$.

${ }^{23}$ CE, 30 décembre 2016, Société Darta c. ACNUSA, n 395681, publié au recueil Lebon, AJDA, 2017, p. 6. 
Après avoir contourné la question dans sa décision Ryan Air du 7 décembre $2005^{24}$, le Conseil d'Etat a finalement explicitement reconnu l'applicabilité de l'article 6 de la Convention EDH dans l'arrêt Compagnie Blue Line du 23 avril $2009^{25}$, transposant ainsi les jurisprudences Didier ${ }^{26}$ et Parent $^{27}$ à la procédure de sanction mise en œuvre par l'ACNUSA. Il distingue ainsi les garanties qui s'appliquent dès la phase administrative de la procédure de celles qui s'appliquent uniquement au stade juridictionnel. Dans ce domaine comme dans d'autres, c'est l'existence d'un recours de plein contentieux, dans le cadre duquel le juge bénéficie de pouvoirs étendus, qui justifie cette distinction dans l'applicabilité des garanties du droit au procès équitable.

Cette reconnaissance de l'applicabilité de l'article 6 de la Convention EDH n'a cependant pas engendré de constats d'inconventionnalité. En outre, depuis que la Cour EDH a clarifié sa position sur la séparation des fonctions dans l'arrêt Grande Stevens du 4 mars $2014^{28}$, il est difficile de contester le manque de séparation des fonctions de poursuite, d'instruction et de

${ }^{24}$ CE, 7 décembre 2005, Ryan Air, n 270424 ; LPA, 8 août 2006, p. 14, concl. Isabelle de Silva. Le Conseil d'Etat ne répond pas à la question de l'applicabilité de l'article 6 Convention EDH à l'ACNUSA alors qu'Isabelle de Silva avait proposé de considérer qu'il s'agissait bien d'accusations en matière pénale et que l'article 6 était applicable, dans les conditions de la jurisprudence Didier.

${ }_{25}$ CE, 23 avril 2009, Compagnie Blue Line, $\mathrm{n}^{\circ} 314918$ : l'article 6 de la Convention EDH est applicable aux sanctions prononcées par l'ACNUSA. Celle-ci décide du bien-fondé d'accusations en matière pénale au sens de l'article 6. Dès lors, même s'il ne s'agit pas d'un tribunal, elle doit respecter certaines des garanties de l'article 6, notamment les droits de la défense. Cela a été ensuite confirmé : CE, 19 mai 2010, Compagnie Majestic executive aviation, $\mathrm{n}^{\circ} 327255$.

${ }^{26}$ CE, ass., 3 décembre 1999, Didier, n² 207434, rec. p. 399 ; RFDA, 2000, p. 584 , concl. Alain Seban.

${ }^{27}$ CE, sect., 27 octobre 2006, Parent, rec. P. 454 ; LPA, 2007, $\mathrm{n}^{\circ} 133$, note Jean-Baptiste Dubrulle.

${ }^{28}$ CEDH, 4 mars 2014, Grande Stevens et a. c/Italie, ${ }^{\circ}$ 18640/10, 18647/10, $18663 / 10,18668 / 10$ et 18698/10 ; RJEP 2014, comm. 48, note G. Eckert. Elle clarifie sa position par rapport à l'arrêt Dubus c. France du 11 juin 2009 (n ${ }^{\circ}$ $5242 / 04$ ) dans lequel elle suggérait la séparation des fonctions de poursuite, d'instruction et de sanction. Dans l'arrêt Grande Stevens et a. c/Italie, la Cour admet que le manque de séparation des fonctions puisse ne pas constituer une violation du droit au procès équitable. En l'espèce, les trois fonctions de poursuite, d'instruction et de sanctions étaient exercées sous l'autorité du Président d'une AAI. Même si cela représente un défaut d'impartialité objective, la Cour n'en déduit pas pour autant la violation de l'article 6 de la convention, étant donné que la décision de sanction peut faire l'objet d'un recours de plein contentieux 
sanction sur le terrain du droit européen des droits de l'homme. Néanmoins, tout n'est peut-être pas encore tout à fait joué dans ce domaine $^{29}$. Sans aller jusqu'à retenir la position la plus exigeante -à savoir la nécessaire séparation des trois fonctions de poursuite, d'instruction et de sanction $^{30}$ - on peut se référer à la position adoptée par le Conseil constitutionnel ${ }^{31}$. Ce dernier a jugé, à propos de l'Autorité de régulation des communications électroniques et des postes, que les dispositions du Code des postes et télécommunications méconnaissaient le principe d'impartialité tiré de l'article 16 de la Déclaration des droits de l'homme et du citoyen de 1789 en ce qu'elles n'assuraient pas «la séparation au sein de l'Autorité entre, d'une part, les fonctions de poursuite et d'instruction des éventuels manquements et, d'autre part, les fonctions de jugement des mêmes manquements $»^{32}$. De ce point de vue, l'organisation de l'ACNUSA ne semble pas satisfaisante, au moins sur un plan théorique. En effet, son Président intervient au niveau des trois fonctions. En ce qui concerne tout d'abord la fonction de poursuite, le Président de l'ACNUSA dispose de l'opportunité des poursuites ${ }^{33}$. En ce qui concerne ensuite la fonction d'instruction, l'article L. 6361-11 du Code des transports prévoit que le Président de l'ACNUSA nomme le rapporteur permanent, lequel a pour mission de superviser l'instruction du dossier ${ }^{34}$. Certes, le pouvoir de direction du Président sur le rapporteur n'est pas explicite, mais il n'est pas non plus exclu, d'autant que l'article L. 6361-14 du Code des transports énonce que le rapport est placé «auprès de l'autorité ». En ce qui concerne enfin la fonction de sanction, le Président de l'ACNUSA siège au sein du collège qui prononce la sanction. Le risque juridique pourrait donc se manifester à l'avenir davantage du côté du droit interne que du côté du droit européen des droits de l'homme.

\footnotetext{
${ }^{29}$ Pour une perspective synthétique quant à l'évolution de la jurisprudence dans ce domaine, v. Hubert Delzangles, «La séparation des fonctions dans la procédure de sanctions du CSA », in Céline Bloud-Rey et Jean-Jacques Menuret (dir.), Le droit de la régulation audiovisuelle et le numérique, Larcier, 2016 , p. 59.

${ }^{30}$ En ce sens, v. Hubert Delzangles, « Un vent d'impartialité souffle encore sur le droit de la régulation », AJDA, 2014, p. 1021.

${ }^{31}$ Cette position a été initiée dans l'arrêt CC, $\mathrm{n}^{\circ}$ 2012-280 QPC du 12 octobre 2012, Société Groupe canal Plus et a., § 21. Elle est explicite dans l'arrêt CC, $\mathrm{n}^{\circ}$ 2013-331 QPC du 5 juillet 2013, Société Numéricable SAS et a., § 12.

${ }^{32} \mathrm{CC}, \mathrm{n}^{\circ}$ 2013-331 QPC du 5 juillet 2013, Société Numéricable SAS et a., $\S 12$.

${ }^{33}$ Article L. 6361-14 al. 2 du Code des transports.

${ }^{34}$ Article R. 227-1 s. du Code de l'aviation civile.
} 
Sous réserve de cette dernière question, dès lors que le législateur, le pouvoir réglementaire et l'ACNUSA assurent le respect des garanties procédurales et limitent ainsi le nombre d'annulations, leur mise en œuvre ne nuit pas à l'efficacité du système de répression. En revanche, la question du contrôle de la proportionnalité de la sanction est plus importante car elle peut influencer plus directement le niveau de dissuasion du système.

\section{B. LE CONTROLE DE LA PROPORTIONNALITE DES SANCTIONS PAR LE JUGE ADMINISTRATIF}

Le contrôle de proportionnalité des sanctions est susceptible d'impacter leur efficacité dans la mesure où il peut influencer leur sévérité et donc leur caractère dissuasif ${ }^{35}$. Comme dans d'autres domaines, le juge administratif contrôle la proportionnalité de l'amende à la gravité de l'infraction commise ${ }^{36}$. A ce titre, le juge $\mathrm{du}$ fond exerce un contrôle normal d'adéquation. Il peut évidemment utiliser son pouvoir de réformation et abaisser le montant de l'amende, ce qu'il ne s'est pas privé de faire à plusieurs reprises ${ }^{37}$. Quel est le modus operandi du juge pour

35 Il faut par ailleurs noter que le juge administratif a jusqu'ici systématiquement refusé d'admettre la force majeure. Les divers éléments invoqués par les compagnies aériennes pour justifier leurs infractions, que ce soit la présence d'un avion gênant le décollage (CE, 13 juillet 2010, Société Air France, $\mathrm{n}^{\circ}$ 334748), de mauvaises conditions météorologiques ayant retardé l'arrivée des agents de piste (CE, 7 mai 2008, Société Air France, n ${ }^{\circ}$ 305826) ou la grève des contrôleurs aériens (CE, 21 juin 2010, Société Air France, 328891), n'ont jamais été considéré par le juge comme étant irrésistibles. En effet, ces différents éléments n'ont pas pour effet de contraindre les appareils à décoller et, ainsi, à commettre une infraction. Néanmoins, s'il rejette ces motifs dans le cadre de l'appréciation de la force majeure, il les examine dans le cadre du contrôle de la proportionnalité de la sanction. Il met en balance les raisons invoquées par les compagnies avec l'importance des nuisances engendrées

${ }^{36}$ CE, 23 avril 2009, Compagnie Blue-Line, n ${ }^{\circ} 314918$; CE, 18 décembre 2009, Compagnie Blue-Line, $\mathrm{n}^{\circ} 323460$; CE, 19 mai 2010, Compagnie Majestic executive aviation, $\mathrm{n}^{\circ} 327255$.

${ }^{37}$ CE, 23 avril 2009, Société Air France, n 312824 ; Revue de droit des transports, 2009, comm. 158, note Vincent Correia («ce dépassement, de vingt-trois minutes, trouve son origine dans la nécessité de débarquer un passager dont l'état d'ébriété ne s'était pas manifesté au moment de l'embarquement $\gg)$; CE, 29 octobre 2009, Société Air France, $\mathrm{n}^{\circ}$ 310613 (empiètement de 16 minutes sur la plage horaire de nuit en raison de mauvaises conditions météorologiques ayant conduit le contrôle aérien à 
procéder à ce contrôle ? L'examen particulier de la proportionnalité de la sanction débute toujours par un rappel du montant maximal de l'amende encourue. Le juge insiste ensuite d'une part sur les nuisances induites par le manquement -en quelque sorte en guise d'éléments à charge- et, d'autre part, sur les circonstances que l'ACNUSA a pris en considération pour moduler le montant de l'amende, à titre d'éléments à décharge. Dans la grande majorité des décisions, il en déduit l'absence de disproportion, ou il réforme la décision et diminue l'amende. Quoi qu'il en soit, le juge administratif vérifie que l'ACNUSA a bien examiné les circonstances particulières du manquement ${ }^{38}$ pour apprécier le montant de l'amende.

La façon dont le juge exerce son contrôle nous semble de nature à préserver le caractère dissuasif du système répressif mis en place dans ce domaine : l'exclusion des faits justificatifs et la prise en compte mesurée des circonstances particulières invoquées par les compagnies en témoignent.

En effet, certains faits justificatifs sont purement et simplement exclus par le juge. Autrement dit, ils ne sauraient justifier que la compagnie aérienne ne soit pas sanctionnée. Il en va tout d'abord ainsi des aléas habituels rencontrés par tout transporteur aérien. Dans l'arrêt Ryan Air du 7 décembre 2005 ${ }^{39}$, le Conseil d'Etat refuse d'admettre que certains aléas inhérents à l'activité de tout transporteur aérien tels que les conditions météorologiques, les retards accumulés dans d'autres aéroports ou les problèmes liés au contrôle aérien puissent l'exonérer d'une sanction ${ }^{40}$. En effet, comme l'exprimait Isabelle de Silva dans ses conclusions, «si l'on devait admettre que ce type de motif suffit à faire obstacle au

retarder 170 vols); CAA Paris, 20 juin 2006, Royal Air Maroc, $\mathrm{n}^{\circ}$ 05PA04740 ; TA Paris, 18 juin 2013, Société El Al Lignes aériennes d'Israël, $\mathrm{n}^{\circ} 1211632 / 2-1$ (en l'espèce le juge tient compte de l'importance du caractère dissuasif des sanctions dans son contrôle de proportionnalité : " $a u$ vu de l'ensemble de ces circonstances et afin de tenir compte de la nécessité de conférer aux sanctions qu'elle prononce un caractère dissuasif, en infligeant à la requérante une amende d'un montant de 18000 euros alors que le montant maximum des sanctions que l'autorité peut prononcer est de 20000 euros pour une personne morale, l'ACNUSA n'a pas pris à l'encontre de la société El Al une sanction disproportionnée ») ; TA Paris, 12 novembre 2014, Société Enter Air, no 1405189 (v. le rapport d'activités 2014 de l'ACNUSA, p. 69).

${ }^{38}$ CE, 29 janvier 2010, Société Air France, n 327068.

${ }^{39}$ CE, 7 décembre 2005, Ryan Air, n 270424 ; LPA, 8 août 2006, p. 14, concl. Isabelle de Silva.

${ }^{40}$ En revanche, certains de ces aléas sont susceptibles d'être pris en compte pour minimiser le montant de l'amende (v. infra). 
prononcé d'une sanction, tout le dispositif deviendrait vain, car un transporteur pourrait presque toujours s'exonérer de l'amende en invoquant ces considérations. (...) Tout le pari du dispositif est d'obliger les transporteurs à prévoir, dans la prévision et le déroulement des vols, une "marge de sécurité" horaire suffisante pour éviter que les vols n'atterrissent au cour de la nuit ${ }^{41}$. Ensuite, dans le même arrêt, le Conseil d'Etat rejette deux moyens d'ordre économique tendant à faire admettre le manquement. La compagnie Ryan Air invoquait l'impact excessif des sanctions sur la rentabilité de ses vols ${ }^{42}$. Là aussi, la position d'Isabelle de Silva est stricte. Selon elle, «cette circonstance, en elle-même, ne démontre pas une illégalité de la sanction : le dispositif ayant un but dissuasif, les montants infligés ne peuvent être trop faibles, car si tel était le cas les compagnies n'auraient aucune incitation à modifier l'organisation de leurs vols pour respecter la réglementation $\gg{ }^{43}$. De plus, la compagnie expliquait qu'elle était contrainte de faire décoller ses appareils durant les plages horaire de nuit afin d'éviter d'annuler le vol et de devoir ainsi indemniser les passagers en application d'un règlement européen ${ }^{44}$. Enfin, le juge a considéré qu'un écart de trajectoire par rapport au volume de protection environnementale ne pouvait pas être justifié en l'espèce par les conditions météorologiques défavorables dont le pilote était informé et qu'il était en mesure de parer ${ }^{45}$. L'examen de la jurisprudence montre ainsi que le juge administratif, en

41 Isabelle de Silva, conclusions sur CE, 7 décembre 2005, Ryan Air, $\mathrm{n}^{\circ} 270424$; LPA, 8 août 2006, p. 14.

${ }^{42}$ Selon les conclusions d'Isabelle de Silva, «la société indique qu'un vol ne lui rapporterait qu'un chiffre d'affaires moyen de $6.000 €$ : dans ce cadre, les sanctions infligées, même si elles n'atteignent pas le maximum de $12.000 €$, amputeraient très sérieusement les bénéfices attendus par la compagnie » (Isabelle de Silva, conclusions sur CE, 7 décembre 2005, Ryan Air, $\mathrm{n}^{\circ} 270424$; LPA, 8 août 2006, p. 14).

${ }^{43}$ Isabelle de Silva, conclusions sur CE, 7 décembre 2005, Ryan Air, n ${ }^{\circ}$ 270424 ; LPA, 8 août 2006, p. 14.

${ }^{44}$ Une obligation d'assistance aux passagers découle du règlement européen $n^{\circ} 261 / 2004$ du 11 février 2004. Le coût de cette obligation peut aller jusqu'à 600 euros par passager. Selon Vincent Correia, «le calcul économique pour les compagnies aériennes dans une telle situation se révèlera extrêmement simple : lorsque les coûts engendrés par le report au lendemain du vol seront supérieurs au montant prévisible de l'amende, ils n'hésiteront sans doute pas à décoller durant la plage horaire de nuit» (Vincent Correia, «Le contrôle par le Conseil d'Etat de la proportionnalité des sanctions infligées par l'ACNUSA », note sous CE, 30 mars 2009, Cie WDL Aviation GmbH \& Co, $\mathrm{n}^{\circ} 311045$; 23 avril 2009, Société Air France, $n^{\circ} 312824$; Revue de droit des transports, 2009, comm. 158).

${ }^{45}$ CE, 16 novembre 2007, Compagnie aérienne Iberia Lineas, $n^{\circ} 289184$. 
adoptant une position ferme sur ces types de faits justificatifs, tend à préserver l'efficacité du système répressif mis en œuvre par l'ACNUSA.

En revanche, cela ne veut pas dire que le juge ne tient pas compte d'un certain nombre de circonstances particulières dans l'appréciation de la proportionnalité. Ainsi, l'ACNUSA peut légalement tenir compte, à décharge de la compagnie, de certaines de ces circonstances. Tout d'abord, certaines sont prises en considération par l'ACNUSA pour réduire le montant de l'amende. Par exemple, l'ACNUSA a pu légalement tenir compte du fait que l'infraction a été commise en raison d'un mouvement de grève dans les services de contrôle aérien ${ }^{46}$, du remplacement d'une sonde ${ }^{47}$, du remplacement d'une vanne d'air sur un moteur ${ }^{48}$, de la présence d'un autre aéronef gênant le départ $^{49}$, des circonstances météorologiques défavorables ${ }^{50}$, de la vérification technique rendue nécessaire par une odeur de brûlé dans la cabine de l'avion ${ }^{51}$, de la fermeture d'un terminal de l'aéroport ${ }^{52}$, d'une panne sur une passerelle de l'aéroport ${ }^{53}, \mathrm{du}$ nécessaire débarquement d'un enfant malade ${ }^{54}$ ou bien encore de l'arrivée tardive de l'équipe de football des Girondins de Bordeaux retenue à l'issue du match qu'elle venait de jouer par les forces de l'ordre pour des raisons de sécurité ${ }^{55}$. Ensuite, l'ACNUSA peut légalement tenir compte de la bonne volonté de la compagnie, laquelle se manifeste par exemple à travers sa politique de remplacement des appareils les plus bruyants ${ }^{56}$. Enfin, la faible gravité du manquement est susceptible de réduire le montant de l'amende. Ainsi, l'ACNUSA peut légalement tenir compte du fait que l'écart avec l'horaire limite était peu important $^{57}$, que l'aéronef soit peu bruyant ${ }^{58}$, de la faible ampleur

\footnotetext{
${ }^{46}$ CE, 7 décembre 2005, Ryan Air, n 270424 ; LPA, 8 août 2006, p. 14, concl. Isabelle de Silva.

${ }^{47}$ CE, 7 mai 2008, Société Air France, n 305814.

${ }^{48}$ CE, 7 mai 2008, Société Air France, $n^{\circ} 305822$.

${ }^{49} \mathrm{CE}, 13$ juillet 2010, Société Air France, $\mathrm{n}^{\circ} 334748$.

${ }^{50}$ CE, 7 mai 2008, Société Air France, $n^{\circ} 305826$.

${ }^{51}$ CE, 7 mai 2008, Société Air France, $n^{\circ} 305823$.

${ }^{52}$ CE, 29 octobre 2008, Société Air France, $n^{\circ} 312816$.

${ }^{53}$ CE, 7 mai 2008, Société Air France, $n^{\circ} 305825$.

${ }^{54}$ CE, 7 mai 2008, Société Air France, $n^{\circ} 305824$.

${ }^{55}$ CE, 5 novembre 2009, Société Blue Line, n 313430.

${ }^{56}$ CE, 2 juillet 2008, Société Air France ; Revue de droit des transports, 2008, comm. 245, obs. Loïc Grard.

${ }^{57}$ CE, 7 mai 2008, Société Air France, n 305821 ; CE, 7 mai 2008, Société Air France, $n^{\circ} 305823$; CE, 7 mai 2008, Société Air France, n 305826 ; CE,
} 
de la déviation de trajectoire par rapport au volume de protection environnementale ${ }^{59}$ ou encore du fait que le survol ait eu lieu en début d'après-midi à une heure moins gênante pour les riverains ${ }^{60}$. L'examen de la jurisprudence montre dès lors que même si l'ACNUSA tient compte de tous ces éléments, cela ne la conduit pas à prononcer des amendes trop faibles. Elle tient compte des circonstances particulières sans annihiler l'effet dissuasif de l'amende.

In fine, le contrôle exercé par le juge est ferme en ce qui concerne les faits justificatifs invoqués par les compagnies aériennes mais il est en même temps suffisamment souple pour permettre à l'ACNUSA de tenir compte des circonstances afin de moduler le montant de l'amende. Dès lors, la façon dont le juge administratif exerce le contrôle de proportionnalité nous semble plutôt adaptée. Certes, on peut déplorer l'inévitable effet de substitution que produit le contrôle de proportionnalité. En effet, à travers le contrôle de proportionnalité, le juge devient le véritable régulateur des nuisances aériennes ${ }^{61}$. Néanmoins, cette critique n'est pas propre à ce domaine de régulation.

Si l'on se place sous l'angle de l'efficacité du contrôle des nuisances, le contrôle de proportionnalité pose néanmoins un autre type de problème, qui demanderait une étude à lui tout seul.

Le contrôle de proportionnalité est en pratique un contrôle à sens unique. Autrement dit, il n'est susceptible de jouer qu'en faveur d'une réduction de l'amende, au bénéfice de la compagnie aérienne. En effet, les possibilités d'obtenir, au contentieux, l'aggravation d'une sanction administrative sont très étroites. D'une part, une sanction ne peut pas être aggravée en appel. Il s'agit de l'interdiction de la reformation in pejus. Cette « règle

18 décembre 2008, Société Air France, $\mathrm{n}^{\circ}$ 312821, Revue de droit des transports, 2009, comm. 79, note Sébastien Martin ;

${ }^{58} \mathrm{CE}, 30$ mars 2009, Cie WDL Aviation GmbH \& Co, ${ }^{\circ}$ 311045, Revue de droit des transports, 2009, comm. 158, note Vincent Correia ; CE, 29 janvier 2010, Société Air France, $n^{\circ} 327068$.

${ }^{59}$ CE, 23 avril 2009, Compagnie Blue Line, $\mathrm{n}^{\circ} 314919$.

${ }^{60} \mathrm{CE}, 19$ mai 2010, Compagnie Majestic executive aviation, $\mathrm{n}^{\circ} 327255$.

${ }^{61}$ « Le juge exerce un contrôle qui le mène à des considérations d'opportunité qui le placent en régulateur judiciaire de la politique de lutte contre les nuisances sonores en provenance des aéronefs commerciaux» (Loïc Grard, «Proportionnalité des amendes prononcées par l'ACNUSA : le Conseil d'Etat régulateur des nuisances sonores aériennes », note sur CE, 2 juillet 2008, SA Air France, Revue de droit des transports, 2008, comm. 245). 
essentielle qui domine l'ensemble du droit pénal, civil et disciplinaire ${ }^{62}$ est également valable en contentieux administratif ${ }^{63}$ et découle du fait que l'appel ne saurait préjudicier à l'appelant. D'autre part, il résulte de la jurisprudence constitutionnelle l'interdiction faite au juge du plein contentieux d'aggraver la sanction lorsqu'il utilise son pouvoir de réformer la sanction: «le droit de recours étant réservé à la personne sanctionnée, son exercice ne peut, conformément aux principes généraux du droit, conduire à aggraver sa situation » ${ }^{64}$. Néanmoins, cette dernière interdiction n'est pas absolue ${ }^{65}$. L'interdiction d'aggravation ne s'applique que lorsque le recours contre la sanction est le fait de la personne sanctionnée. Elle ne s'applique pas lorsque le recours est effectué par une autre personne. En atteste la recevabilité du recours formé par le Président de l'Autorité des marchés financiers ${ }^{66}$. Cela renvoie ainsi à la question de l'intérêt à agir des tiers à l'encontre des sanctions administratives, intérêt dont le juge retient une

${ }^{62}$ Rémi Keller, «Les pouvoirs du juge d'appel en matière disciplinaire », conclusions sur CE, 17 juillet 2013, M. Dioum, $\mathrm{n}^{\circ}$ 362481, RFDA, 2013, p. 1183.

${ }^{63}$ Sur ce point, voir les conclusions de Rémi Keller, « Les pouvoirs du juge d'appel en matière disciplinaire », conclusions sur CE, 17 juillet 2013, M. Dioum, $\mathrm{n}^{\circ}$ 362481, RFDA, 2013, p. 1183.

${ }^{64} \mathrm{CC}, \mathrm{n}^{\circ}$ 88-248 DC du 17 janvier 1989, CSA, § 31 : «Considérant qu'il convient de relever également que toute décision infligeant une sanction peut faire l'objet devant le Conseil d'État d'un recours de pleine juridiction, comme le précise l'article $42-8 ;(\ldots)$; que le droit de recours étant réservé à la personne sanctionnée, son exercice ne peut, conformément aux principes généraux du droit, conduire à aggraver sa situation ». Décision confirmée par CC, 25 février 1992, n 92-307 DC ; CE, sect., 23 novembre 2001, Compagnie nationale Air France, $\mathrm{n}^{\circ} 195550$.

${ }^{65}$ Par ailleurs, s'il est clair que le juge du plein contentieux ne peut pas aggraver la sanction, qu'en est-il de l'autorité administrative elle-même dans l'hypothèse où la première sanction prononcée est simplement annulée par le juge ? Lorsque l'autorité administrative statue de nouveau sur le dossier, peutelle adopter une sanction plus sévère ? Ce cas n'est, à notre connaissance, pas encore tranché.

${ }^{66}$ V. CE, 3 février 2016, Président de l'AMF, n 369198 : le président de l'Autorité des marchés financiers demandait au Conseil d'Etat de réformer la décision de la commission des sanctions de cette autorité en prononçant des sanctions plus sévères. La requête est rejetée par le Conseil d'Etat car celui-ci estime que les sanctions ne sont pas insuffisamment proportionnées, autrement dit qu'elles étaient suffisamment sévères. Néanmoins, le Conseil d'Etat ne mentionne pas l'interdiction faite au juge d'aggraver les sanctions dans le cadre de son pouvoir de réformation. En effet, comme le précise explicitement la décision du Conseil constitutionnel du 17 janvier 1989 ( ${ }^{\circ}$ 88-248 DC), cette interdiction ne s'applique qu'en cas de recours effectué par la personne sanctionnée. Sur recours d'une autre personne, l'aggravation des sanctions est théoriquement possible. 
conception assez étroite ${ }^{67}$, comme du reste à l'égard d'un refus de sanctionner ${ }^{68}$.

Pour autant, sous cette dernière réserve, le contrôle du juge administratif est plutôt adapté et finalement relativement neutre. Ce qui semble véritablement déterminant est l'organisation institutionnelle du contrôle des nuisances. De ce point de vue, le recours à une autorité indépendante constitue un modèle à reproduire.

\section{LE RECOURS À UNE AUTORITÉ INDEPENDANTE, UN MODĖLE À REPRODUIRE}

Même si le contrôle exercé par l'ACNUSA n'a évidemment pas réglé tous les problèmes de nuisances et de pollutions liés aux activités aéroportuaires ${ }^{69}$, son niveau de dissuasion apparaît plutôt satisfaisant lorsqu'on le compare à la situation d'autres domaines du droit de l'environnement (A). Il tend ainsi à faire figure de modèle à reproduire, ce qui conduit à expliciter les raisons pour lesquelles la création d'une autorité publique indépendante dans le domaine de l'environnement pourrait être pertinente (B).

\footnotetext{
${ }^{67}$ En matière de sanctions disciplinaires, v. CE, sect., 13 janvier 1950, Union générale des fédérations de fonctionnaires, rec. p. 26 ; 17 mai 2006, Bellanger, $\mathrm{n}^{\circ}$ 268938, rec. p. 257 ; AJDA, 2006, p. 1513, concl. Remi Keller. En ce qui concerne les sanctions administratives non disciplinaires, la décision Tinez a ouvert la voie à un élargissement sur la question de l'intérêt à agir des tiers (CE, sect., 30 novembre 2007, Michel Tinez et a. contre ACAM, ${ }^{\circ} 293952$, AJDA, 2007, p. 2285). Damien Botteghi résume la situation dans ses conclusions sur l'arrêt Syndicat CFE-CGC France Télécom du 8 avril 2013 (CE, 8 avr. 2013, $\mathrm{n}^{\circ}$ 359789, RJEP, 2013, comm. 42, concl. Damien Botteghi) : en l'absence de texte organisant l'accès des tiers au déclenchement des sanctions, la jurisprudence se rapproche de l'appréciation classique de l'intérêt à agir qui consiste à rechercher un intérêt direct et certain (v. Damien Botteghi, «Intérêt à agir dans une procédure répressive », conclusions sur CE, 8 avril 2013, Syndicat CFE-CGC France Télécom, n 359789, RJEP, 2013, comm. 42).

${ }^{68}$ CE, sect., 10 juillet 1995, Mme Laplace, rec. p. 302 : un professeur n'a pas intérêt à agir à l'encontre du refus du proviseur d'exercer des poursuites contre un élève. V. Hubert Delzangles, «Le refus de sanctionner », JCP A, 2013, n 2078.

69 L'examen de cette question nécessiterait des compétences dépassant largement celles du juriste. Il ne s'agit pas ici d'affirmer que les pollutions et nuisances ont été réduites grâce au contrôle exercé par l'ACNUSA -il serait trop difficile de le démontrer scientifiquement- mais seulement de souligner l'intérêt de son architecture institutionnelle.
} 


\section{A. UN MODELE COMPARATIVEMENT INTERESSANT}

Trois critères peuvent être utilisés afin d'évaluer -du moins de porter une appréciation- sur le dispositif répressif mis en œuvre par l'ACNUSA : le niveau de détection des infractions, l'intervention effective de la sanction et sa proportionnalité. Ces éléments permettent de se faire une idée du caractère dissuasif du système $^{70}$. Les chiffres regroupés dans le tableau ci-dessous, extraits des rapports annuels de l'ACNUSA, fournissent des données utiles à l'appréciation de ces critères.

Chiffres relatifs au pouvoir répressif de l'ACNUSA ${ }^{71}$

\begin{tabular}{|l|c|c|c|c|}
\hline & 2012 & 2013 & 2014 & 2015 \\
\hline Nombre de PV & 490 & 418 & 393 & 413 \\
\hline $\begin{array}{l}\text { Nombre de décisions prises } \\
\text { au titre du pouvoir de } \\
\text { sanction (dossiers instruits) }\end{array}$ & 439 & 413 & 387 & 335 \\
\hline $\begin{array}{l}\text { Nombre de manquements } \\
\text { n'ayant pas fait l'objet d'une } \\
\text { amende }\end{array}$ & $\begin{array}{c}120 \\
(27,3 \%)\end{array}$ & $\begin{array}{c}68 \\
(16,5 \%)\end{array}$ & $\begin{array}{c}97 \\
(25,1 \%)\end{array}$ & $\begin{array}{c}96 \\
(28,7 \%)\end{array}$ \\
\hline $\begin{array}{l}\text { Nombre de manquements } \\
\text { ayant fait l'objet d'une } \\
\text { amende }\end{array}$ & $\begin{array}{c}319 \\
(72,7 \%)\end{array}$ & $\begin{array}{c}345 \\
(83,5 \%)\end{array}$ & $\begin{array}{c}290 \\
(74,9 \%)\end{array}$ & $\begin{array}{c}239 \\
(71,3 \%)\end{array}$ \\
\hline $\begin{array}{l}\text { Montant moyen des } \\
\text { amendes }\end{array}$ & 8694 & 7887 & 8077 & $14033^{72}$ \\
\hline $\begin{array}{l}\text { Montant total des amendes } \\
\text { prononcées }\end{array}$ & 2773500 & 2721050 & 2342200 & 3354000 \\
\hline
\end{tabular}

Tout d'abord, le niveau de détection des infractions ne semble pas excessivement faible, même si certaines difficultés persistent. Entre le début des années 2000 et l'année 2012, le nombre de dossiers instruits annuellement par l'ACNUSA a doublé, passant d'environ $200^{73}$ à environ $400^{74}$. Néanmoins, ces deux dernières années, ce nombre tend à se réduire, atteignant 335 dossiers en 2015. Il en va de même du nombre de PV qui diminue légèrement depuis 2012. Il est cependant très difficile d'interpréter ces chiffres. En effet, la baisse peut être le signe d'une bonne efficacité du système répressif, laquelle entrainant -dans les faits-

\footnotetext{
${ }^{70}$ V. Julien Bétaille, Les conditions juridiques de l'effectivité de la norme..., op. cit., p. 203 s..

${ }^{71}$ Sources : rapports d'activités de l'ACNUSA.

${ }^{72} \mathrm{~A}$ partir du $1^{\text {er }}$ janvier 2014 , le montant maximal est passé de 20000 à 40000 euros.

${ }^{73}$ Entre 2001 et 2004, le nombre de dossiers instruits était de 200 par an en moyenne (Cour des comptes, Les aéroports français face aux mutations du transport aérien, juillet 2008, p. 121).

${ }^{74}$ Tableau, deuxième ligne.
} 
une baisse du nombre de manquements. Cela peut aussi, à l'inverse, être le signe du développement de plus grandes tolérances administratives dans la détection des infractions. Il est donc difficile de se faire une idée claire du niveau de détection. Néanmoins, il semble d'un niveau suffisamment élevé pour alimenter correctement la procédure de répression mise en œuvre par l'ACNUSA.

Le travail de détection mené par la DGAC est cependant parfois critiqué dans divers rapports. Outre que celle-ci ne consacrerait pas suffisamment de moyens humains à cette tâche ${ }^{75}$, il est surtout relevé que les agents de la DGAC auraient par le passé pris l'habitude de se mettre d'accord pour ne pas relever certains manquements des compagnies aériennes. Cette situation est relevée tant par le CGEDD $^{76}$ que par l'ACNUSA ${ }^{77}$, les associations $^{78}$ et les parlementaires. Ces derniers remarquent ainsi que « la DGAC est donc juge et partie et opère elle-même un tri préalable alors que ce devrait être le rôle de l'ACNUSA ${ }^{79}$. En effet, en procédant ainsi, elle s'attribue le pouvoir d'opportunité des poursuites qui est pourtant confié par la loi au Président de l'ACNUSA ${ }^{80}$. Elle vient en outre tarir, à la source, la procédure de sanction. Il est difficile de savoir si cette pratique des agents de la DGAC est toujours d'actualité aujourd'hui. Néanmoins, cela ne serait le cas échéant pas très surprenant car il s'agit d'une pratique assez fréquente dans le domaine de la répression, qu'elle soit pénale ou administrative, singulièrement dans le domaine de l'environnement.

Ensuite, pour que le dispositif répressif soit véritablement dissuasif, il est nécessaire que la «réponse administrative » soit la plus systématique possible. Autrement dit, en cas de manquement -lorsque ce dernier est constaté- une sanction doit effectivement intervenir, même si son niveau de sévérité est

\footnotetext{
${ }^{75}$ Conseil général de l'environnement et du développement durable, Prise en compte de l'environnement par la direction générale de l'Aviation civile, rapport, février 2012, p. 43.

${ }^{76}$ Ibidem, p. 56.

${ }_{78}^{77}$ Rapport d'activités de l'ACNUSA 2014, p. 62.

78 Selon l'association Avevy, «loin de recenser la totalité des cas de manquements, ces agents opèrent une sélection de cas qu'ils vont instruire et transmettre à l'ACNUSA pour poursuites» (v. Jacques Alain Bénisti et Christophe Bouillon, Rapport d'information sur les nuisances aéroportuaires, op. cit., pp. 236-237).

${ }^{79}$ Jacques Alain Bénisti et Christophe Bouillon, ibidem, pp. 236-237.

${ }^{80}$ Article L. 6361-14 al. 2 du Code des transports.
} 
faible. Cela peut paraître évident. Pourtant, le niveau des tolérances administratives est tellement élevé dans d'autres domaines de la répression environnementale qu'un tel rappel s'avère important. En l'occurrence, pour ce qui concerne le contrôle des nuisances aéroportuaires par l'ACNUSA, le nombre de manquements n'ayant pas fait l'objet d'une amende s'élève à environ un tiers: le taux de réponse administrative aux manquements oscille ainsi entre 71 et $83 \%$ entre 2012 et $2015^{81}$. Dès lors, tous les manquements ne sont pas sanctionnés, le Président de l'ACNUSA exerçant bel et bien son pouvoir d'opportunité des poursuites à l'égard des PV qui sont dressés ${ }^{82}$. Pour autant, le taux de réponse administrative est sans commune mesure avec ce qui existe pour la police des installations classées ou pour la police de l'eau. On passe de $70 \%$ minimum pour les nuisances aéroportuaires à environ $10 \%$ pour les polices de l'eau et des $\mathrm{ICPE}^{83}$. Partant, l'observateur extérieur peut avoir le sentiment que dans les autres domaines, l'administration fait preuve de mansuétude et utilise la moindre opportunité pour ne pas sanctionner. A l'inverse, l'ACNUSA fait une application raisonnée de son pouvoir de sanction. En effet, la plupart des dossiers donnent lieu à une sanction -il existe dans les faits une réponse administrative- mais les circonstances particulières de la commission des manquements sont prises en compte au titre de la proportionnalité de la sanction. Ces éléments révèlent probablement une grande différence de culture administrative ${ }^{84}$ mais surtout, ils suggèrent que la mise en place d'une AAI permet un exercice plus adéquat du pouvoir de sanction.

Enfin, pour ce qui concerne le critère de la proportionnalité des sanctions, il convient d'examiner le niveau des amendes qui sont prononcées. La situation n'est là aussi pas parfaite, mais elle est comparativement beaucoup plus satisfaisante que dans d'autres

\footnotetext{
${ }^{81}$ Quatrième ligne du tableau.

${ }^{82}$ La doctrine de l'ACNUSA sur les classements sans suite est connue. Ceuxci interviennent soit lorsque les circonstances particulières à la commission des faits le justifient, soit lorsque les faits consignés dans le PV ne sont pas constitutifs d'un manquement pouvant donner lieu à sanction (Rapport d'activités de 1'ACNUSA 2015, p. 94).

${ }^{83} \mathrm{~V}$. infra.

${ }^{84}$ L'administration de l'environnement ne manifeste pas beaucoup de volonté à faire de la répression un véritable outil des politiques publiques. Cela a été maintes fois mis en avant. V. notamment Pierre Lascoumes, L'éco-pouvoir, La découverte, 1994 ; Julien Bétaille, «L'efficacité de la répression administrative », in Les sanctions administratives dans les secteurs techniques, CERAP, ISJPS UMR 8103, rapport pour la mission de recherche Droit \& Justice, 2016, p. $121 ; 123$.
} 
domaines. Le caractère pécuniaire de l'amende semble pédagogique en ce sens qu'il permet à la compagnie de comparer facilement l'intérêt qu'il y a à éviter d'être sanctionné ${ }^{85}$ : il s'agit bien évidemment de faire en sorte que le respect de la règle soit plus rentable pour la compagnie que sa violation ${ }^{86}$. Surtout, même si le Président de l'ACNUSA demande un nouveau doublement du montant maximal des amendes ${ }^{87}$, le montant moyen des amendes prononcées par l'ACNUSA ces dernières années est en augmentation. Entre 2012 et 2015, il a presque doublé, notamment grâce à un relèvement du montant maximal de l'amende en 2014. Nous sommes passé de 6600 euros entre 2000 et $2005^{88}$ à 8000 euros en 2012 puis à 14000 euros en 2015. Autrement dit, le montant moyen des amendes a plus que doublé ces dernières années. Alors certes, ce montant peut toujours être considéré comme insuffisant, mais il s'agit indéniablement -compte tenu de ces montants- d'un élément qui ne peut pas être ignoré par les compagnies aériennes.

Cela est d'autant plus vrai que l'ANCUSA veille particulièrement à l'exécution des sanctions qu'elle prononce ${ }^{89}$. Elle a même été jusqu'à imposer l'immobilisation à l'aéroport Charles de Gaulle d'un avion de la compagnie Enter Air au cours de l'été 2016, cela en raison de l'importance des amendes impayées par la

${ }^{85}$ Cela n'est pas le cas lorsque la sanction administrative prend d'autres formes, comme par exemple la plupart des mesures prévues aux articles L. 171-7 et L. 171-8 du Code de l'environnement.

${ }^{86}$ Les conclusions de Isabelle de Silva sur l'affaire Ryan Air fournissent quelques éléments sur cette question. En l'espèce, la compagnie prétendait qu'une amende de 6000 euros annihilait la rentabilité d'un vol (v. Isabelle de Silva, conclusions sur CE, 7 décembre 2005, Ryan Air, n 270424 ; LPA, 8 août 2006, p. 14). Ces éléments sont néanmoins très difficiles à vérifier.

${ }^{87}$ Les amendes infligées par l'ACNUSA sont jugées «insuffisantes » par le rapport parlementaire des députés Jacques Alain Bénisti et Christophe Bouillon. Selon eux, il faut «exclure tout arbitrage de la part des compagnies entre la pénalité et le gain à tirer d'une infraction» (Jacques Alain Bénisti et Christophe Bouillon, Rapport d'information sur les nuisances aéroportuaires, op. cit., p. 123). Selon ce même rapport, le Président de l'ACNUSA, Monsieur Victor Haim, souhaiterait que le montant maximal de l'amende soit encore doublé pour être porté à 80000 euros (ibidem, p. 123).

${ }^{88}$ Cour des comptes, Les aéroports français face aux mutations du transport aérien, juillet 2008, p. 121.

${ }^{89}$ Le président de l'ACNUSA est en charge du recouvrement des amendes. Le niveau de recouvrement est plutôt encourageant selon les éléments mis en évidence par le rapport parlementaire des députés Jacques Alain Bénisti et Christophe Bouillon (v. Jacques Alain Bénisti et Christophe Bouillon, ibidem, p. 125). 
compagnie $^{90}$. Cela a également été le cas le 16 décembre 2016 à l'aéroport Paris - Charles-de-Gaulle concernant un avion de la société Turkménistan Airlines ${ }^{91}$.

En définitive, même si un système répressif peut toujours être amélioré, celui qui est mis en œuvre par l'ACNUSA semble $a$ minima fonctionner correctement, ce qui est loin d'être le cas dans d'autres domaines. Il nous semble que la principale raison de ce bon fonctionnement tient au fait que l'ACNUSA est une autorité administrative indépendante. Elle est ainsi à même d'exercer son action de façon impartiale, ce qui réduit considérablement le niveau de tolérance administrative habituellement constaté dans le domaine de l'environnement. L'ACNUSA constitue ainsi un modèle à reproduire.

\section{B. LA REPRODUCTION DU MODELE : VERS UNE AUTORITE PUBLIQUE INDEPENDANTE ENVIRONNEMENTALE (APIE)}

En premier lieu, quatre raisons conduisent à proposer l'institution d'une autorité publique indépendante environnementale (APIE). La comparaison de l'action de l'ACNUSA avec d'autres domaines montre que l'indépendance favorise l'impartialité et, in fine, l'effectivité de sanctions. C'est la première raison qui conduit à plaider en faveur d'une autorité publique indépendante dans le domaine de l'environnement, même si ce n'est pas la seule. La répression administrative, comme la répression pénale d'ailleurs, se révèle extrêmement faible en matière d'environnement. Nombreux sont les rapports à l'avoir mis en évidence $^{92}$. Par exemple, dans le domaine de l'eau, le rapport du Conseil d'Etat consacré à cette question relevait en 2010 que seulement $8,5 \%$ des manquements faisaient l'objet d'une mise en

\footnotetext{
90 «Un avion immobilisé à cause d'amendes impayées pour nuisances sonores », La Tribune, 11 août 2016.

${ }^{91}$ Selon le site internet de l'ACNUSA, «cette compagnie avait commis 11 manquements, respectivement en 2011 et entre juillet 2014 et janvier 2015. Tous ces manquements, consistant en des décollages irréguliers, avaient été sanctionnés par des amendes pour un montant total de 186000 euros. Cette société restant sourde aux demandes itératives de régler sa dette, l'ACNUSA a dî recourir à la procédure assez lourde de la saisie conservatoire pour le recouvrement d'une somme de 204000 euros correspondant au montant des amendes augmentées de la pénalité de 10 \% pour paiement tardif » (consulté le 3 mars 2017).

92 Conseil d'Etat, L'eau et son droit, Rapport public 2010, vol. 2, La documentation française, 2010 ; Cour des comptes, Rapport public annuel 2010, La documentation française, 2010.
} 
demeure $^{93}$. Dans le domaine des installations classées, en 2014, les contrôles ont abouti à 2280 mises en demeure mais seulement 250 sanctions administratives ont été adoptées ${ }^{94}$. Vu sous un angle optimiste, cela veut dire que plus de $89 \%$ des mises en demeure ont atteint leur objectif de retour à la conformité. Au contraire, cela peut aussi vouloir dire que le niveau des tolérances administratives approche $90 \%$. L'importance des tolérances administratives s'explique par le fait qu'au-delà de problèmes de culture répressive et du « chantage à l'emploi » qui y est pratiqué, l'autorité administrative titulaire du pouvoir de sanction n'a pas assez de distance vis-à-vis des entités régulées. Le préfet est sensible aux intérêts économiques locaux et, de ce fait, est réticent lorsqu'il s'agit de sanctionner. Le positionnement institutionnel du titulaire du pouvoir de sanction revêt donc une grande importance. L'exemple de l'ACNUSA montre que l'institution d'une autorité indépendante permet une plus grande distance vis-à-vis du secteur régulé et ainsi une meilleure répression.

Par ailleurs, l'institution d'une autorité indépendante permet traditionnellement de couper court à un certain nombre de conflits d'intérêts dont peuvent être frappées les autorités administratives classiques. Il s'agit de la deuxième raison en faveur d'une autorité indépendante dans le domaine de l'environnement. Comme le résume Hubert Delzangles, «le pouvoir exécutif intervient en tant qu'opérateur sur les marchés, par l'intermédiaire d'opérateurs appartenant intégralement ou partiellement à l'Etat (...). Il lui est donc délicat, en même temps, de réguler directement ces marchés sauf à se retrouver assez souvent en situation de conflits d'intérêts ${ }^{96}$. Il en va de même

\footnotetext{
93 «Sur les $40 \%$ de contrôles effectués sur le terrain et donnant des résultats non conformes, $91 \%$ débouchent d'après le ministère de l'Écologie sur un simple rappel à la réglementation et $8,5 \%$ seulement sur une mise en demeure » (Conseil d'Etat, L'eau et son droit, op. cit., p. 221).

${ }^{94}$ Les chiffres au 31 décembre 2014 sont disponibles sur le site internet de l'inspection des installations classées : http://www.installationsclassees.developpement-durable.gouv.fr/Chiffres-clede-1-Inspection.html.

${ }^{95}$ La Cour des comptes constatait déjà en 2010 qu'en matière de police de l'eau « $26 \%$ des contrôles réalisés par les services de 1'Etat donnent lieu à une réponse administrative ou pénale, mais seuls $1 \%$ conduisent à une sanction. (En matière d'installations classées), $7 \%$ se traduisent par une sanction administrative ou pénale » (Cour des comptes, Rapport public annuel 2010, op. cit., p. 625).

${ }^{96}$ Hubert Delzangles, «Un vent d'impartialité souffle encore sur le droit de la régulation », AJDA, 2014, p. 1021. L'auteur explique également que l'idée
} 
dans le domaine de l'environnement où l'Etat, à travers le pouvoir exécutif, se retrouve dans la position du régulateur mais est aussi opérateur à travers la figure de l'Etat actionnaire. EDF ou AREVA en sont autant d'exemples. Un préfet ne peut pas, de façon objectivement impartiale, sanctionner, au titre de la législation sur les installations classées par exemple, une entreprise dont l'Etat est actionnaire.

La troisième raison qui milite en faveur de la création d'une autorité indépendante est la nécessité d'éviter les distorsions de concurrence sur le territoire national. En l'état actuel des choses, le même type de manquement n'est pas nécessairement sanctionné de la même façon selon le département dans lequel le manquement est commis. Les tolérances administratives à l'égard de certains industriels conduisent à des situations de concurrence déloyale, comme l'a d'ailleurs admis la Cour de cassation ${ }^{97}$. Le caractère centralisé de la sanction par une autorité nationale permet de déterminer une politique répressive et de l'appliquer de façon uniforme. Cela limite ainsi les distorsions de concurrences suscitées par un contrôle déconcentré. Par exemple, l'action de l'ACNUSA montre que les compagnies aériennes sont sanctionnées de façon uniforme, quel que soit l'aéroport concerné.

La dernière raison -la plus importante et la plus générale- en faveur d'une autorité indépendante est la garantie du droit de l'homme à l'environnement. La création d'autorités publiques indépendantes est souvent justifiée par la protection des droits fondamentaux $^{98}$. Or, le droit à l'environnement est aujourd'hui un droit reconnu tant par la Constitution ${ }^{99}$, que par le droit international $^{100}$ ou la jurisprudence de la Cour européenne des

d'impartialité «empêche ainsi qu'un ministère exerçant un pouvoir de direction sur l'opérateur historique dont il détient en partie le capital, mette ce dernier dans une situation où il serait nécessairement amené à abuser de sa position dominante ».

${ }^{97}$ Pour le juge judiciaire, la violation du droit des installations classées peut constituer un acte de concurrence déloyale (Cass., com., 21 janvier 2014, ${ }^{\circ}$ 12-25443 ; Environnement, 2014, rép. 4, note François-Guy Trébulle : à propos de l'exploitation d'une casse de véhicules sans autorisation).

98 En témoigne les nombreuses AAI qui ont pour mission la protection des droits fondamentaux (par ex. le Défenseur des droits, la CNIL, le CSA, etc.). V. aussi CC, 26 juillet 1984, n 84-173 DC, § 4.

${ }^{99}$ Article $1^{\text {er }}$ de la Charte de l'environnement.

${ }^{100}$ Notamment à l'article $1^{\text {er }}$ de la Convention sur l'accès à l'information, la participation du public au processus décisionnel et l'accès à la justice en matière d'environnement, signée à Aarhus (Danemark) le 25 juin 1998. 
droits de l'homme ${ }^{101}$. Une autorité publique indépendante pourrait ainsi contribuer à renforcer la protection du droit à l'environnement. Sa garantie implique, compte tenu de la sensibilité des problèmes environnementaux, une triple indépendance vis-à-vis de l'Etat, des acteurs privés et, plus généralement, vis-à-vis des intérêts du présent. Cette autorité indépendante pourrait ainsi veiller spécifiquement à la prise en considération des enjeux du long terme ${ }^{102}$.

En deuxième lieu, cette nouvelle autorité indépendante créée dans le domaine de l'environnement pourrait assumer trois types de missions, le point commun entre ces trois missions étant que chacune nécessite une indépendance accrue. Il s'agirait de certaines missions consultatives, de la mission de police et de répression ainsi que vis-à-vis de l'indemnisation du préjudice écologique pur.

Tout d'abord, l'APIE pourrait assurer une mission consultative auprès des pouvoirs publics et, en particulier, dans le cadre des procédures d'évaluation environnementale. D'une part, et de façon assez classique, l'autorité pourrait être sollicitée pour avis par les pouvoirs publics sur les domaines relevant de son champ de compétence, à savoir la protection de l'environnement. D'autre part, l'APIE pourrait donner son avis dans le cadre des procédures d'évaluation environnementale ${ }^{103}$, en lieu et place des actuelles «autorités environnementales » ${ }^{104}$. Le besoin d'indépendance est ici particulièrement important. Il s'agit de donner un avis sur la qualité de l'évaluation environnementale

${ }^{101}$ V. Cour EDH, 27 janvier 2009, Tatar c. Roumanie, RJE, 2010, p. 62, note Jean-Pierre Marguénaud.

${ }^{102}$ Sur cette question, v. Dominique Bourg et al., Pour une $6^{\mathrm{e}}$ République écologique, Odile Jacob, 2011.

${ }^{103}$ Il s'agit des études d'impact environnemental des projets (article L. 122-1 s. du Code de l'environnement) et des évaluations environnementales des plans et des programmes (article L. 122-4 s. du Code de l'environnement). Le rapport du groupe de travail présidé par Yves Jegouzo se prononce en faveur d'une autorité indépendante chargée de l'«évaluation et (du) contrôle des évaluations environnementales » (Yves Jegouzo et al., Pour la réparation du préjudice écologique, Rapport du groupe de travail installé par Madame Christiane Taubira, 17 septembre 2013, p. 25 ). Le même rapport propose que l'autorité assure également une "garantie de mise en ouvre et respect des principes d'information et de participation ». Nous ne sommes pas favorables à ce que l'APIE assure cette dernière mission car elle pourrait plutôt être confiée à la Commission nationale du débat public, autorité administrative indépendante déjà compétente en matière de participation du public.

104 Il s'agit de l'autorité environnementale nationale et des autorités environnementales régionales prévues aux articles L. $122-3$ II. $4^{\circ}$ et R. 122-6 s. du Code de l'environnement. 
qui soit tout à la fois indépendant du pétitionnaire et de l'auteur de l'évaluation d'une part, et de l'autorité administrative en charge de la décision d'autre part. Surtout, confier cette mission à l'APIE permettrait de se mettre définitivement à l'abri de la critique née à l'occasion de l'arrêt Seaport rendu par la CJUE le 20 octobre $2011^{105}$.

Ensuite, l'APIE disposerait d'une mission répressive: elle se verrait confier l'ensemble des pouvoirs de sanction administrative existant dans le domaine de l'environnement ${ }^{106}$, principalement en lieu et place des préfets. Plus largement, elle regrouperait l'ensemble des inspecteurs de l'environnement disséminés notamment entre les inspections des installations classées, l'Agence pour la biodiversité (AFB) ${ }^{107}$ et l'Office national de la chasse et de la faune sauvage (ONCFS). Ainsi, elle serait chargée de constater les infractions administratives et pénales dans le domaine de l'environnement, ce qui impliquerait évidemment pour elle de définir une politique répressive. Elle exercerait directement le pouvoir de sanction administrative, le juge judiciaire demeurant compétent en matière pénale, sur la base des constatations effectuées par les agents de l'APIE.

${ }^{105}$ CJUE, 20 octobre 2011, Seaport, C-474/10, RJE, 2012, p. 184, note Olivier Vidal ; Droit européen de l'environnement - Jurisprudence commentée, 3 ème éd., Larcier, p. 204, note Marc Clément. Cet arrêt, s'il n'impose pas à proprement parler la création d'une autorité indépendante, implique une séparation fonctionnelle au sein de l'entité administrative entre les fonctions de consultation et de décision. Cette séparation suppose notamment que l'autorité en charge de donner un avis sur l'évaluation environnementale «soit pourvue de moyens administratifs et humains qui lui sont propres, et soit ainsi en mesure de remplir les missions confiées aux autorités de consultation » (CE, 26 juin 2015, Association France Nature Environnement, $n^{\circ}$ 360212). Plus récemment, dans un jugement rendu le 2 novembre 2016, le tribunal administratif d'Orléans a constaté que, au niveau local, «c'est bien la même autorité (...) qui a exercé la compétence consultative en matière environnementale et la compétence visant à la délivrance de l'autorisation attaquée ». Dès lors, l'ancien article R. 122-6 du Code de l'environnement est contraire au droit de l'Union européenne car l'autorité qui dispose d'une compétence consultative en matière d'environnement ne dispose pas d'une « autonomie effective » (TA Orléans, 2 novembre 2016, Association Boischaut Marche environnement et a., $\mathrm{n}^{\circ}$ 1500442, Droit de l'environnement, 2016, p. 407).

${ }^{106}$ V. les articles L. 171-6 s. du Code de l'environnement. V. Chantal Cans, «La réforme, tant attendue, du droit répressif de 1'environnement », Droit Administratif, 2013, ét. 1.

107 Sur cette agence, v. Agathe Van Lang, «La loi Biodiversité du 8 août 2016 : une ambivalence assumée - Le droit renouvelé : la reprise des procédés efficaces $-2^{\mathrm{e}}$ Partie », AJDA, 2016, p. 2492. 
Enfin, l'APIE aurait un rôle important à jouer au niveau de la réparation du préjudice écologique et de la compensation. En ce qui concerne la réparation du préjudice écologique, le législateur a récemment décidé d'ouvrir cette action en réparation «à toute personne ayant qualité et intérêt à agir, telle que l'Etat, l'Agence française pour la biodiversité, les collectivités territoriales et leurs groupements dont le territoire est concerné, ainsi que les établissements publics et les associations agréées ou créées depuis au moins cinq ans à la date d'introduction de l'instance qui ont pour objet la protection de la nature et la défense de l'environnement ${ }^{108}$. Cette disposition fait dès lors de ces personnes les bénéficiaires des dommages et intérêts susceptibles d'être versés au titre de la réparation du préjudice écologique pur $^{109}$. Si le choix du législateur avait été de limiter le bénéfice de cette action à l'Etat, ce que nous appelions de nos vœux ${ }^{110}$, alors cela aurait suscité des problèmes particuliers sur le plan de la fonction de sanction de la responsabilité administrative ${ }^{111}$. Dans ce cadre, une APIE aurait pu avoir un rôle déterminant à jouer pour préserver cette fonction de sanction ${ }^{112}$. Néanmoins, même si le choix du législateur a été différent, la création d'une APIE pourrait être utile à plus d'un titre. A l'instar de ce que proposait le rapport rendu au garde des sceaux en septembre 2013 par un

\footnotetext{
${ }^{108}$ Article 1248 du Code civil.

${ }^{109}$ L'article 1249 du Code civil dispose néanmoins que «La réparation $d u$ préjudice écologique s'effectue par priorité en nature ». Il ajoute qu' «en cas d'impossibilité de droit ou de fait ou d'insuffisance des mesures de réparation, le juge condamne le responsable à verser des dommages et intérêts, affectés à la réparation de l'environnement, au demandeur ou, si celui-ci ne peut prendre les mesures utiles à cette fin, à l'Etat ».

${ }^{110}$ V. Julien Bétaille, Les conditions juridiques de l'effectivité de la norme..., op.cit., $\mathrm{n}^{\circ}$ 417. Si les éléments de l'environnement «font partie du patrimoine commun de la nation » tel que l'affirme l'article L. 110-1 du Code de l'environnement, alors la nation, personnifiée par l'Etat, est la véritable victime du préjudice écologique pur. Dès lors, l'Etat devrait être le seul bénéficiaire des indemnisations versées au titre de la réparation de ce préjudice.

${ }^{111}$ Cela supposerait évidemment au préalable que le juge administratif accepte à son tour de réparer le préjudice écologique pur. Sur ce point, v. Julien Bétaille, Les conditions juridiques de l'effectivité de la norme..., op.cit., $\mathrm{n}^{\circ}$ 411 s. ; Christian Huglo, «L'inéluctable prise en compte du dommage écologique par le juge administratif », AJDA, 2013, p. 667 ; «La notion de réparation du préjudice écologique à l'épreuve du droit administratif », Energie - Environnement - Infrastructures, 2016, ét. 21 ; Marthe Lucas, «Préjudice écologique et responsabilité - Pour l'introduction légale du préjudice écologique dans le droit de la responsabilité administrative », Environnement, 2014, ét. 6.

${ }^{112}$ V. Julien Bétaille, Les conditions juridiques de l'effectivité de la norme..., op.cit., $\mathrm{n}^{\circ} 418$ s..
} 
groupe de travail présidé par Yves Jegouzo, une autorité indépendante pourrait avoir pour mission la «surveillance des atteintes à l'environnement par le constat des préjudices écologiques, la réalisation ou la supervision des expertises et la saisine du juge de la réparation ${ }^{113}$. Ce serait d'ailleurs cette mission qui justifierait de doter l'autorité de la personnalité juridique $^{114}$. En ce qui concerne la compensation, l'APIE pourrait jouer un rôle d'expertise pour évaluer la crédibilité et la suffisance des mesures de compensation proposées par les pétitionnaires. Elle assurerait également une fonction de régulateur des marchés de compensation appelée de ses vœux par Gilles J. Martin ${ }^{115}$.

En dernier lieu, la création d'une APIE aurait des conséquences importantes sur le paysage institutionnel de l'environnement actuel. Les missions actuellement dévolues aux autorités environnementales, au niveau national comme en région, seraient transférées à l'APIE. Cela impliquerait la création d'antennes régionales de l'APIE. En outre, l'APIE remplacerait deux autorités administratives indépendantes: l'ACNUSA et l'Autorité de sureté nucléaire $(\mathrm{ASN})^{116}$. Elle absorberait

\footnotetext{
${ }^{113}$ Yves Jegouzo et al., Pour la réparation du préjudice écologique, Rapport du groupe de travail installé par Madame Christiane Taubira, 17 septembre 2013, p. 25 ; v. Recueil Dalloz, 2013, p. 2347, comm. Gilles J. Martin. Selon les auteurs de ce rapport, la «Haute autorité environnementale " «evrait être dotée de la personnalité juridique et pourrait notamment saisir directement le juge ou relayer, si elle l'estime justifié (compte tenu de sa capacité d'expertise), les requêtes émanant notamment des lanceurs d'alerte au sens de la loi du 16 avril 2013 et préfinancer des mesures d'expertise comme le fait parfois l'Autorité de la concurrence » (rapport p. 26).

114 Sur les autorités publiques indépendantes, v. Sébastien Martin, «Les autorités publiques indépendantes : réflexions autour d'une nouvelle personne publique », RDP, 2013, p. 53.

115 Gilles J. Martin, «Quelle(s) régulation(s) dans l'hypothèse d'un recours aux mécanismes de marché pour protéger l'environnement ? », in Marie-Pierre Camproux-Duffrène et Jochen Sohnle (dir.), Marché et environnement, Bruylant, 2014, p. 476.

116 La troisième AAI existant dans le domaine de l'environnement, la Commission nationale du débat public, dépourvue de mission de sanction et dotée d'une mission très spécifique, demeurerait séparée.

Outre que l'indépendance de l'Autorité de sureté nucléaire soit perfectible (v. Hubert Delzangles, «L'indépendance de l'autorité de sureté nucléaire, des progrès à envisager », Revue juridique de l'environnement, 2013, p. 7), son action en matière de sanction n'est pas convaincante, du moins sur un plan statistique. Ainsi, en 2015, l'ASN n'est parvenue à prendre que 8 «mesures administratives » (Rapport de l'ASN sur l'état de la sûreté nucléaire et de la radioprotection en France en 2015, p. 22-23) alors qu'elle a effectué 1882 inspections et qu'elle a relevé dans ce cadre 1722 «évènements significatifs »,
} 
également les missions de police et de sanction de l'ONCFS comme de l'AFB.

En définitive, le contrôle des nuisances aéroportuaires exercé par l'ACNUSA s'avère particulièrement fertile, y compris sur le plan des idées. Il inspire et justifie la création d'une autorité publique indépendante en matière d'environnement ${ }^{117}$. Cette autorité serait à même d'appliquer, avec toute l'indépendance nécessaire, le droit de l'environnement et de contribuer à la réalisation du droit de l'homme à l'environnement reconnu depuis 2005 par la Constitution française.

dont 1157 événements classés sur l'échelle internationale des évènements nucléaires, comprenant notamment 127 de niveau 1 ou 2 sur l'échelle internationale des évènements nucléaires (ibidem, p. 146), étant entendu que le niveau 0 de cette échelle manifeste déjà un écart par rapport à la règle et que cette échelle compte 7 échelons, le $7^{\text {ème }}$ étant l'accident majeur.

117 La création, sous des formes variables, d'une autorité «indépendante » dans le domaine de l'environnement a été soutenue en doctrine: Julien Bétaille, Les conditions juridiques de l'effectivité de la norme..., op. cit., 2012, n 418 ; Hubert Delzangles, «Les autorités de régulation indépendantes de marché et la prise en compte de l'environnement», in Marie-Pierre Camproux-Duffrène et Jochen Sohnle (dir.), Marché et environnement, Bruylant, 2014, p. 449 ; Gilles J. Martin, «Quelle(s) régulation(s) dans l'hypothèse d'un recours aux mécanismes de marché pour protéger l'environnement? », op. cit., p. 476 ; Agathe Van Lang, «Droit répressif de l'environnement : perspectives en droit administratif », RJE, ${ }^{\circ}$ spécial, 2014, p. 45 ; Yves Jegouzo et al., Pour la réparation du préjudice écologique, op. cit., p. 25 . 